Supporting Information

\title{
Direct Preparation of *MRE Zeolite with Ultra-large Mesoporosity: Strategy \& Working Mechanism
}

Wen Liu ${ }^{a, b}$, Junjie Li*a, Zhenni Liu ${ }^{a}$, Zhiqiang Yang ${ }^{c}$, Xuebin Liu ${ }^{c}$, Xingzhi Tan ${ }^{c}$, Longya Xu ${ }^{a}$, Xiujie Li*a, Xiangxue Zhu*a

aState Key Laboratory of Catalysis, Dalian Institute of Chemical Physics, Chinese Academy of Sciences, 457 Zhongshan Road, Dalian 116023, China.

bUniversity of Chinese Academy of Sciences, Beijing 100049, China.

'Energy Innovation Laboratory, BP office (Dalian Institute of Chemical Physics), Dalian, 116023, China.

*Corresponding author: Junjie Li, lijj@dicp.ac.cn

Xiujie Li, xiujieli@dicp.ac.cn

Xiangxue Zhu, zhuxx@dicp.ac.cn

Dalian Institute of Chemical Physics

Chinese Academy of Sciences

Dalian 116023, P. R. China

Tel/Fax: +86-411-84379279 


\section{Ferrierite zeolite synthesis}

The raw materials used included silica sol $\left(30.57 \mathrm{wt} \% \mathrm{SiO}_{2}, 0.33 \mathrm{wt} \% \mathrm{Na}_{2} \mathrm{O}\right.$, 0.03wt $\% \mathrm{Al}_{2} \mathrm{O}_{3}, 69.07$ wt $\% \mathrm{H}_{2} \mathrm{O}$, Qingdao Haiyang Chemical Co., Ltd.), sodium aluminate solution (homemade, $15.5 \mathrm{wt} \% \mathrm{Na}_{2} \mathrm{O}, 16.8 \mathrm{wt} \% \mathrm{Al}_{2} \mathrm{O}_{3}$ ), sodium hydroxide (96wt \%, Sinopharm Chemical Reagent Co., Ltd), ethylenediamine (En,99wt \%), Tetramethylammonium hydroxide (TMAH, 25wt \% in water, SACHEM) and deionized water (home-made).

FER zeolite was synthesized by hydrothermal route. The molar composition of the initial gel is $0.175 \mathrm{Na}_{2} \mathrm{O} / 0.033 \mathrm{Al}_{2} \mathrm{O}_{3} / 1 \mathrm{SiO}_{2} / 0.18 \mathrm{En} / 14 \mathrm{H}_{2} \mathrm{O} / 0.05 \mathrm{TMAH}$. In a typical synthesis, $0.90 \mathrm{~g} \mathrm{NaOH}, 3.21 \mathrm{~g}$ sodium aluminate solution and $2.89 \mathrm{~g}$ TMAH solution were dissolved in $7.78 \mathrm{~g} \mathrm{H}_{2} \mathrm{O}$, then $1.71 \mathrm{~g}$ En and $31.31 \mathrm{~g}$ silica sol were added in turn. After stirring for $2 \mathrm{~h}$ at $35^{\circ} \mathrm{C}$, the gel was transferred to a $100 \mathrm{ml}$ stainless steel autoclave, and crystallized at $160{ }^{\circ} \mathrm{C}$ in rotation mode $(60 \mathrm{r} / \mathrm{min})$. After crystallization, the autoclave was quenched to room temperature by cool water. Then the suspension was centrifuged, and the solid product was washed with deionized water before drying overnight. The obtained samples with different amounts of TMAH were labeled as FER- $x \mathrm{~T}$ ( $x$ represents the molar ratio of $\mathrm{TMAH} / \mathrm{SiO}_{2}$ ). Conventional FER zeolite (denoted as FER-C) was synthesized in the absence of TMAH, the initial gel is $0.35 \mathrm{OH}^{-} / 0.033 \mathrm{Al}_{2} \mathrm{O}_{3} / 1 \mathrm{SiO}_{2} / 0.18 \mathrm{En} / 14 \mathrm{H}_{2} \mathrm{O}$. 


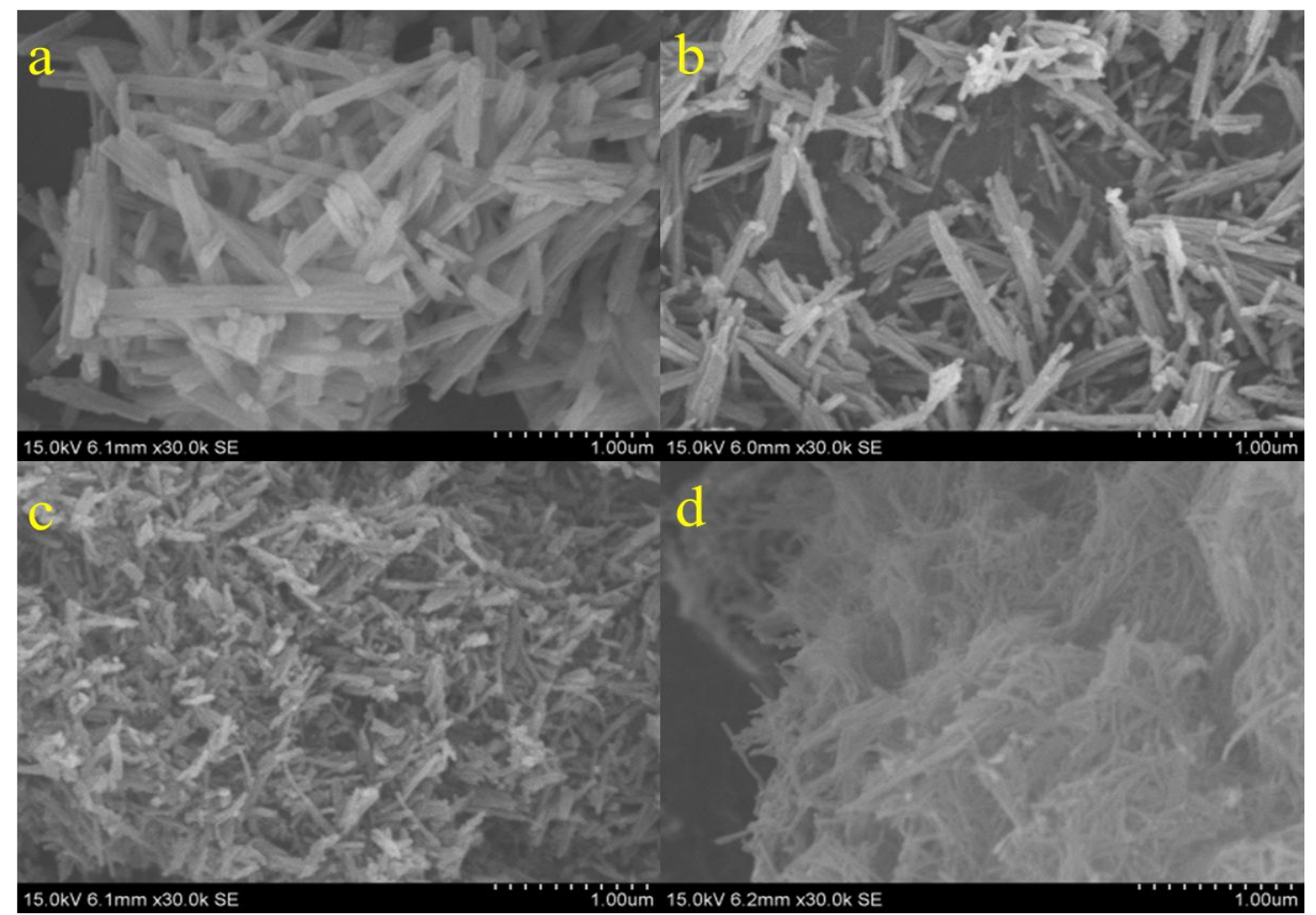

Figure S1. SEM images of (a) MRE-C, (b)MRE-0.05T, (c)MRE-0.1T, (d)MRE-0.15T

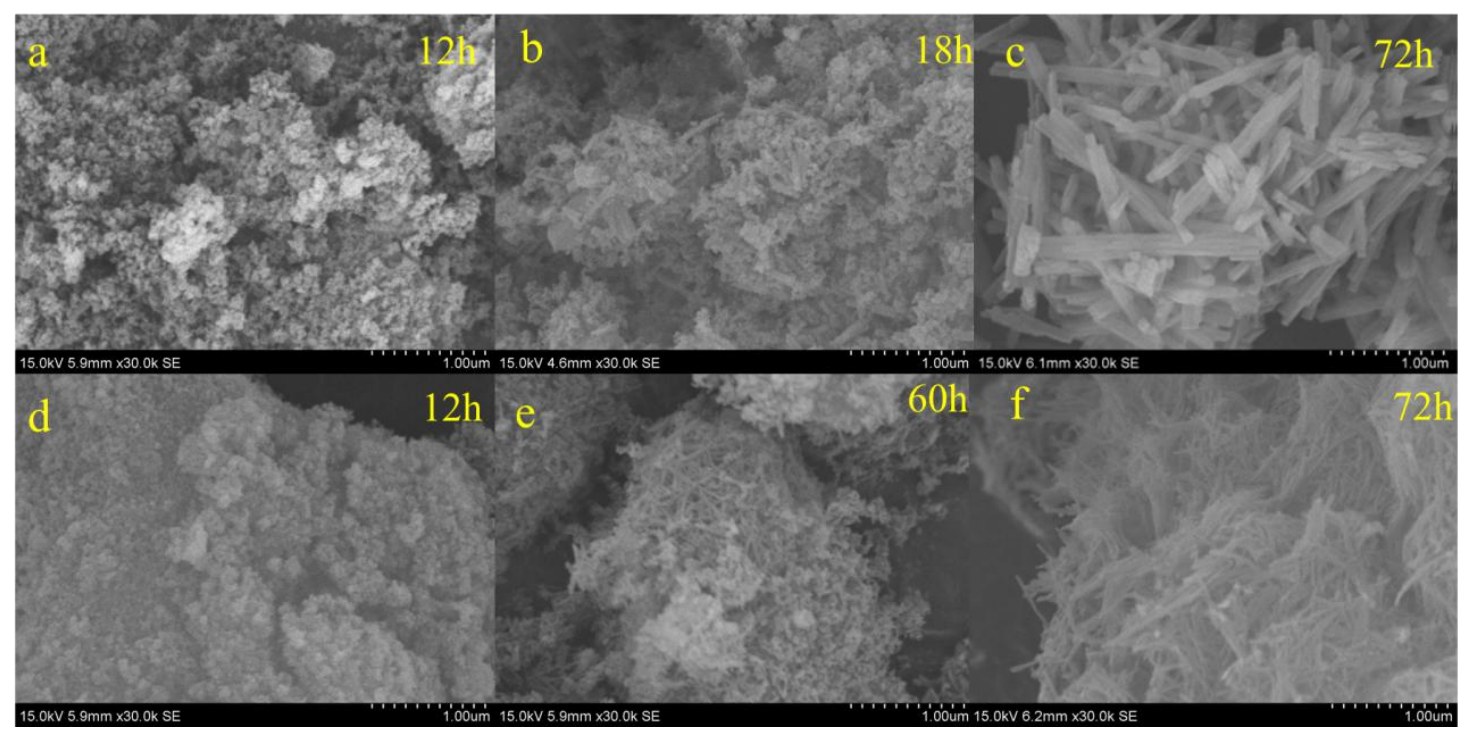

Figure S2. SEM images of samples after crystallization time for different time: $(\mathrm{a} \sim \mathrm{c})$ MRE-C, (d f) MRE-0.15T 


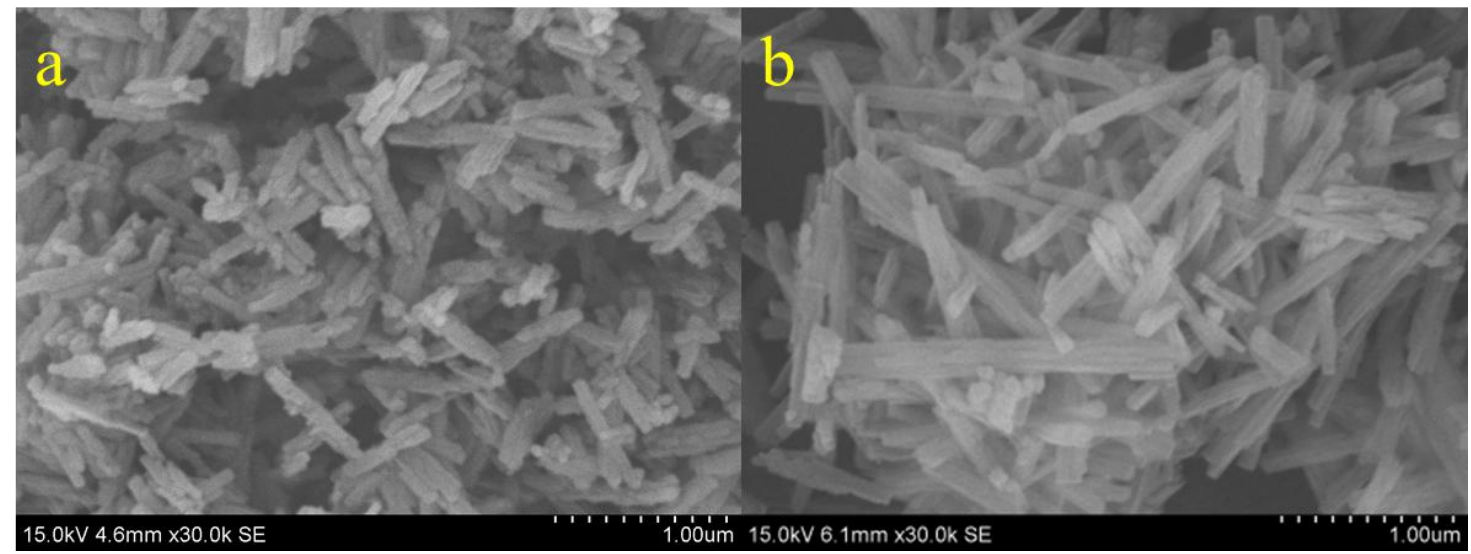

Figure S3. SEM images of (a) MRE-0.15NaBr, (b) MRE-C

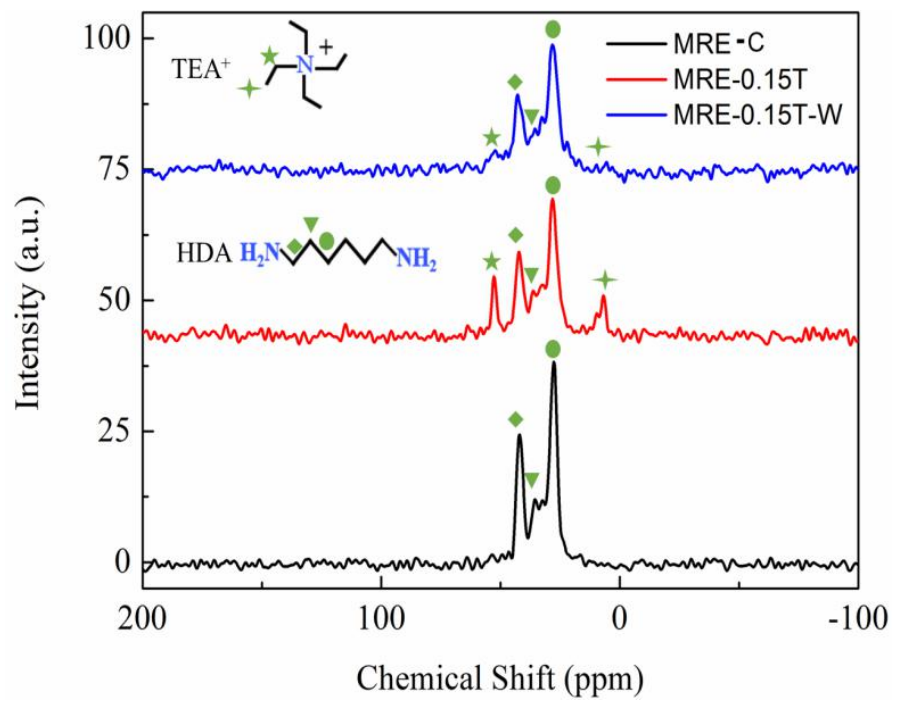

Figure S4. ${ }^{13} \mathrm{C} \mathrm{CP} / \mathrm{MAS}$ NMR spectra of different ZSM-48 samples
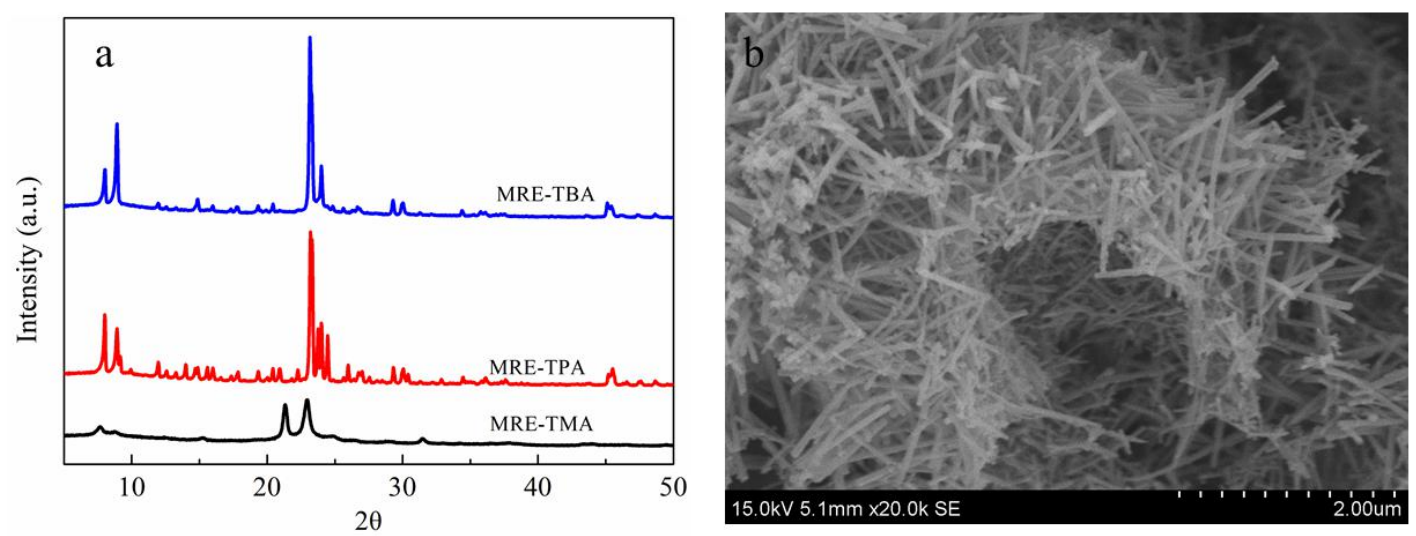

Figure S5. (a) XRD patterns of MRE-TMA, MRE-TPA and MRE-TBA and (b) SEM image of MRE-TMA 


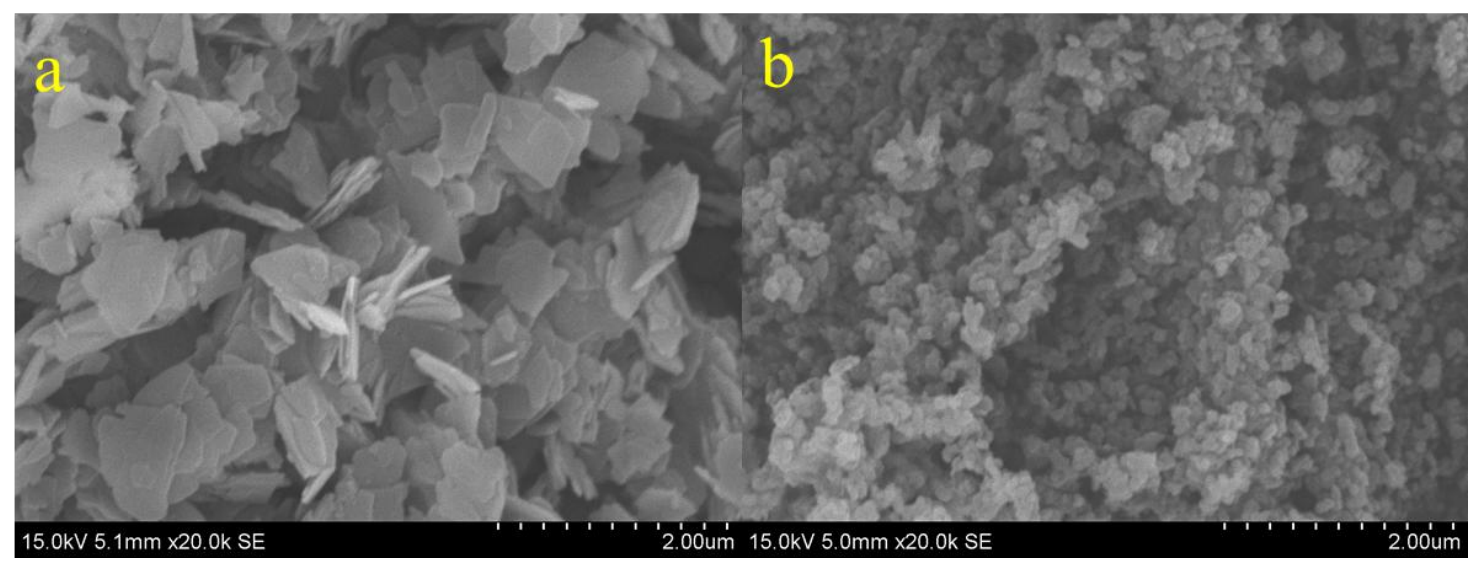

Figure S6. SEM images of FER samples with different TMAH contents: (a) FER-C, (b) FER-0.05T
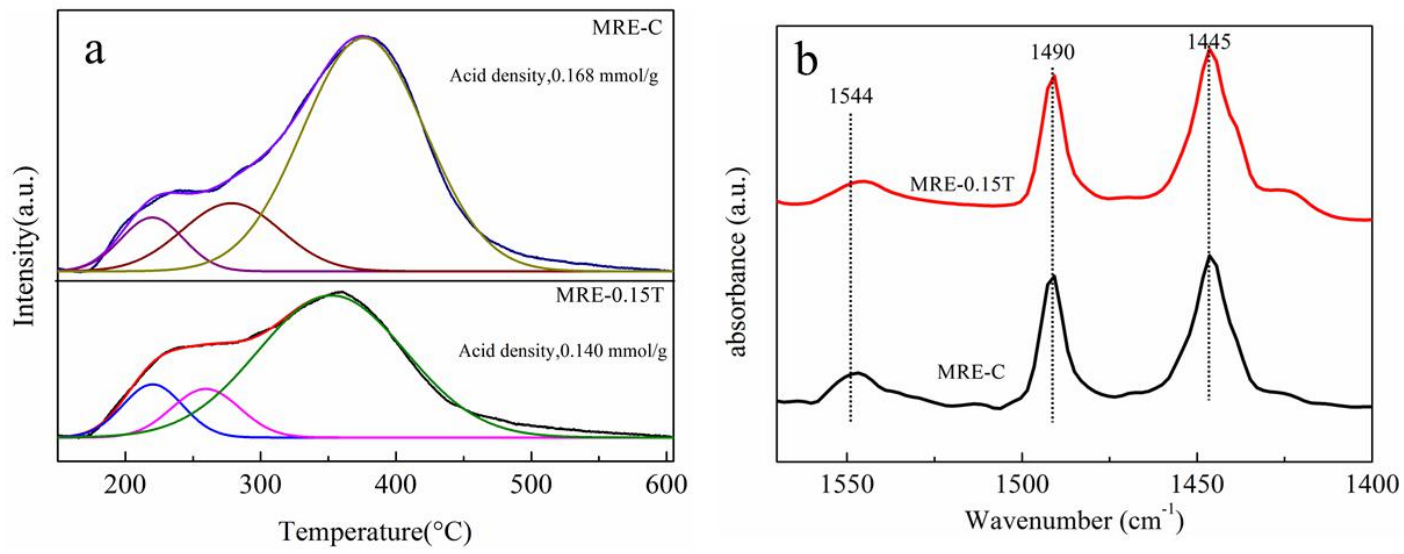

Figure S7. (a) $\mathrm{NH}_{3}$-TPD and (b) Py-IR profiles of MRE-C and MRE-0.15T

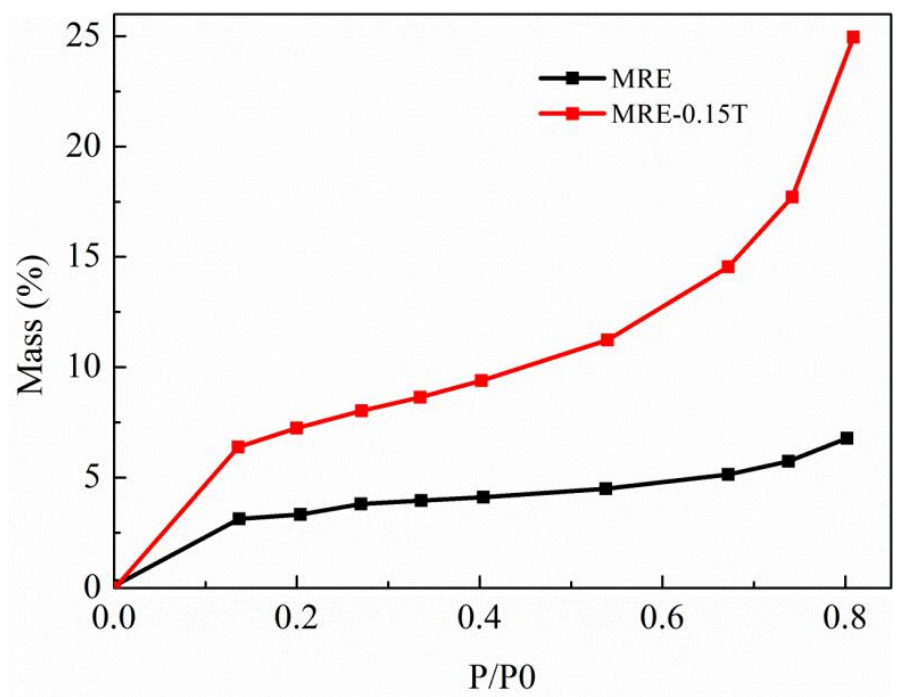

Figure S8. Adsorption isotherms of MX on MRE-0.15T and MRE-C at $30^{\circ} \mathrm{C}$ 
Table S1. The textural properties of hierarchical ZSM-48 zeolites in literatures.

\begin{tabular}{|c|c|c|c|c|c|}
\hline \multirow[t]{2}{*}{ OSDA } & \multirow[t]{2}{*}{ Strategy } & \multicolumn{3}{|c|}{ Texture properties of products } & \multirow[t]{2}{*}{ Reference } \\
\hline & & $\mathrm{S}_{\mathrm{BET}}$ & $\mathrm{V}_{\text {mic }} / \mathrm{cm}^{3} \cdot \mathrm{g}^{-1}$ & $\mathrm{~V}_{\mathrm{meso} /} / \mathrm{cm}^{3} \cdot \mathrm{g}^{-1}$ & \\
\hline${ }^{\mathrm{a}} \mathrm{HMBr}$ & post-treatment & -- & 0.043 & 0.0127 & Muraza et. al. ${ }^{1}$ \\
\hline HDA & post-treatment & 251 & 0.05 & 0.40 & Liang et. al. ${ }^{2}$ \\
\hline${ }^{\mathrm{b}} \mathrm{C}_{22} \mathrm{~N}_{6}$ & in-situ synthesis & 380 & -- & $0.47^{t}$ & Ryong et. al. ${ }^{3}$ \\
\hline 'BPT & in-situ synthesis & 244 & 0.05 & 0.19 & Che et. al. ${ }^{4}$ \\
\hline HDA & in-situ synthesis & 340 & 0.06 & 0.97 & This work \\
\hline
\end{tabular}

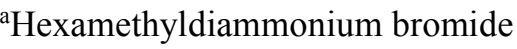

${ }^{b} \mathrm{C}_{22} \mathrm{H}_{45}-\mathrm{N}^{+}\left(\mathrm{CH}_{3}\right)_{2}-\mathrm{C}_{6} \mathrm{H}_{12}-\mathrm{N}^{+}\left(\mathrm{CH}_{3}\right)_{2}-\mathrm{CH}_{2}-\left(\mathrm{C}_{6} \mathrm{H}_{4}\right)-\mathrm{CH}_{2}-\mathrm{N}^{+}\left(\mathrm{CH}_{3}\right)_{2}-\mathrm{C}_{6} \mathrm{H}_{12}-\mathrm{N}^{+}\left(\mathrm{CH}_{3}\right)_{2}-\mathrm{CH}_{2}-\left(\mathrm{C}_{6} \mathrm{H}_{4}\right)$

$\left.-\mathrm{CH}_{2}-\mathrm{N}^{+}\left(\mathrm{CH}_{3}\right)_{2}-\mathrm{C}_{6} \mathrm{H}_{12}-\mathrm{N}^{+}\left(\mathrm{CH}_{3}\right)_{2}-\mathrm{C}_{22} \mathrm{H}_{45}\right]\left(\mathrm{Br}^{-}\right)_{2}\left(\mathrm{Cl}^{-}\right)_{4}$

${ }^{\mathrm{c}}\left(\mathrm{C}_{6} \mathrm{H}_{2}\right)_{3}-\left(\mathrm{O}-\mathrm{C}_{10} \mathrm{H}_{20}-\mathrm{N}^{+}\left(\mathrm{CH}_{3}\right)_{2}-\mathrm{C}_{6} \mathrm{H}_{12}-\mathrm{N}\left(\mathrm{CH}_{3}\right)_{2}\left(\mathrm{Br}^{-}\right)\right)_{6}$

${ }^{\mathrm{t}}$ Total pore volume, Only total pore volume was reported.

Table S2. Quantitative analysis of TG results for ZSM-48 samples

\begin{tabular}{ccccc}
\hline \multirow{2}{*}{ Samples } & \multicolumn{4}{c}{ Weight loss (wt\%) } \\
\cline { 2 - 5 } & $40 \sim 200^{\circ} \mathrm{C}$ & $200 \sim 320^{\circ} \mathrm{C}$ & $320 \sim 800^{\circ} \mathrm{C}$ & $\begin{array}{c}\text { Total } \\
\text { weight Loss }\end{array}$ \\
\hline MRE-C & 0.57 & & 6.58 & 7.15 \\
MRE-0.15T & 0.98 & 4.81 & 7.48 & 13.27 \\
MRE-0.15T-W & 0.73 & 1.34 & 8.04 & 10.11 \\
\hline
\end{tabular}

Table S3. Textural properties of FER-C and FER-0.05T

\begin{tabular}{ccccc}
\hline Samples & ${ }^{\mathrm{a}} \mathrm{S}_{\mathrm{BET}} / \mathrm{m}^{2} \cdot \mathrm{g}^{-1}$ & ${ }^{\mathrm{b}} \mathrm{V}_{\text {micro }} / \mathrm{cm}^{3} \cdot \mathrm{g}^{-1}$ & ${ }^{\mathrm{b}} \mathrm{S}_{\mathrm{ext}} / \mathrm{m}^{2} \cdot \mathrm{g}^{-1}$ & ${ }^{\mathrm{b}} \mathrm{V}_{\text {meso }} / \mathrm{cm}^{3} \cdot \mathrm{g}^{-1}$ \\
\hline FER-C & 403 & 0.13 & 70 & 0.17 \\
FER-TMA & 407 & 0.13 & 72 & 0.33 \\
\hline
\end{tabular}

${ }^{a}$ BET method. ${ }^{b}$ t-plot method

Table S4. SAR and acid distribution of MRE-C and MRE-0.15T

\begin{tabular}{ccccc}
\hline Sample & ${ }^{\mathrm{a} S A R}$ & ${ }^{\mathrm{b}} \mathrm{C}_{\mathrm{B}}(\mathrm{mmol} / \mathrm{g})$ & ${ }^{\mathrm{b}} \mathrm{C}_{\mathrm{L}}(\mathrm{mmol} / \mathrm{g})$ & $\mathrm{C}_{\mathrm{B}} / \mathrm{C}_{\mathrm{L}}$ \\
\hline MRE-C & 187 & 0.034 & 0.082 & 0.41 \\
MRE-0.15T & 194 & 0.026 & 0.097 & 0.27 \\
\hline
\end{tabular}

a: determined from XRF; b: determined from Py-IR 
Table S5. Textural properties of samples before and after reaction

\begin{tabular}{ccccc}
\hline Samples & ${ }^{\mathrm{a}} \mathrm{S}_{\mathrm{BET}} / \mathrm{m}^{2} \cdot \mathrm{g}^{-1}$ & ${ }^{\mathrm{b}} \mathrm{V}_{\text {micro }} / \mathrm{cm}^{3} \cdot \mathrm{g}^{-1}$ & ${ }^{\mathrm{b}} \mathrm{S}_{\mathrm{ext}} / \mathrm{m}^{2} \cdot \mathrm{g}^{-1}$ & ${ }^{\mathrm{b}} \mathrm{V}_{\text {meso }} / \mathrm{cm}^{3} \cdot \mathrm{g}^{-1}$ \\
\hline MRE-C & 254 & 0.07 & 72 & 0.14 \\
MRE-C-used & 45 & 0.005 & 37 & 0.13 \\
MRE-0.15T & 343 & 0.06 & 196 & 0.94 \\
MRE-0.15T-used & 160 & 0.017 & 128 & 0.81 \\
\hline
\end{tabular}

${ }^{a}$ BET method. ${ }^{b}$ t-plot method.

\section{References}

(1) Ahmed, M. H. M.; Muraza, O.; Nakaoka, S.; Jamil, A. K.; Mayoral, A.; Sebastian, V.; Yamani, Z. H.; Masuda, T., Stability Assessment of Regenerated Hierarchical ZSM-48 Zeolite Designed by Post-Synthesis Treatment for Catalytic Cracking of Light Naphtha. Energy Fuels 2017, 31 (12), 14097-14103.

(2) Zhang, M.; Li, C.; Chen, X.; Chen, Y.; Liang, C., Hierarchical ZSM-48-Supported Nickel Catalysts with Enhanced Hydroisomerization Performance of Hexadecane. Ind. Eng. Chem. Res. 2019, 58 (43), 19855-19861.

(3) Kim, W.; Kim, J.-C.; Kim, J.; Seo, Y.; Ryoo, R., External Surface Catalytic Sites of Surfactant-Tailored Nanomorphic Zeolites for Benzene Isopropylation to Cumene. ACS Catal. 2013, 3 (2), 192-195.

(4) Zhang, Y.; Ma, Y.; Che, S., Synthesis of Lamellar Mesostructured ZSM-48 Nanosheets. Chem. Mater. 2018, 30 (6), 1839-1843. 